\title{
Frequency andClinical Presentation of Oral Submucous Fibrosis
}

\author{
Dr.KarunaNooni \\ Assistant Professor OfOtorhinolaryngology, Osmania General Hospital,Hyderabad,India
}

\begin{abstract}
Background: Oral submucous fibrosis (OSMF) is a chronic, precancerous condition of oral mucosa characterized by juxta-epithelial inflammatory reaction along with hyalinization of lamina propria. These alterations causes fibrosis and stiffness of oral mucosa leading to limited mouth opening and related problems. Objective: To evaluate the frequency and clinical presentations of Oral Submucous fibrosis.

Methods: A retrospective study conducted on patients attended to E N T outpatient department at Osmania General Hospital from January 2013 to December 2015. Patients with OSMF irrespective of age and gender were the inclusion criteria. The clinical presentations of patients were tabulated and analyzed by descriptive statistics.

Results: A total number of 70 patients with OSMF were evaluated. The most commonage group was 11-25 years in the study. There were 42 male and 28 female patients with male female ratio of 1.5:1The most common complaint of OSMF patients was burning sensation in oral cavity followed by decreasedmouth opening,recurrent oral ulceration and dryness of mouth. The majority of patients presented with an advanced stage of disease with grade 3 inter-incisal distance.

Conclusion: OSMF is becoming a disease of younger adults with a slight male predilection. The majority of patients presents with a progressive and advanced form of disease.
\end{abstract}

Keywords: OSMF, Precancerous Condition, Fibrosis, Inter-incisal Distance.

\section{Introduction}

In 1952, Schwartz ${ }^{1}$ described five Indian women from Kenya with a condition of the oral mucosa including the palate and pillars of the fauces, which he called "atrophiaidiopathica (tropica) mucosaeoris". Later it was termed oral submucous fibrosis (OSMF) ${ }^{2}$; other names are "diffuse oral submucous fibrosis", "idiopathic scleroderma of the mouth", "idiopathic palatal fibrosis", "sclerosingstomatitis" and"juxta-epithelial fibrosis"3. Submucous fibrosis is an insidious, chronic disease affecting any part of the oral cavity and sometimes the pharynx ${ }^{4}$. Occasionally it is preceded by and/or associated with vesicle formation ${ }^{5}$ and is always associated with a juxta-epithelial inflammatory reaction followed by progressive hyalinization of the lamina propria ${ }^{6}$. The later subepithelial and submucosalmyofibrosis leads to stiffness of the oral mucosa and deeper tissues with progressive limitation in opening of the mouth and protrusion of the tongue, thus causing difficulty in eating, swallowing and phonation ${ }^{7}$. Epithelial atrophy is marked in advanced stages of the disease. Apparent divergencies in these characteristics between groups of patients in different studies raised the question whether OSMF should be considered as one, or more than one disease. Although the evidence that it predisposes to cancer is not yet absolutely conclusive, it is highly probable that this relationship exists. The WHO definition ${ }^{8}$ for an oral precancerous condition-a generalized pathological state of the oral mucosa associated with a significantly increased risk of cancer-accords well with the characteristics of OSMF.

The onset of OSMF is insidious and early symptoms are burning sensation in the mouth with blister formation and ulceration. Moreover, in later stages of disease, the patient presents with limited mouth opening due to stiffening of oral mucosa leading to difficulty in eating, swallowing, speech and maintenance of oral hygiene. Clinically, the mucosa appears blanched and opaque and fibrous bands are palpable in the buccal mucosa. More et $\mathrm{al}^{9}$. proposed a new classification to stage the severity of the disease on basis of clinical presentation. According to his study, blanching of oral mucosa was categorized as stage 1, presence of palpable fibrous bands in buccal mucosa and pharynx was graded as stage 2 and palpable bands involving multiple oral sites was documented as stage 3 . However, presentation of OSMF along with other malignant disorders or oral carcinoma was recorded as stage 4 . Moreover, he further presented the functional staging of disease based on different mouth opening range. The inter-incisal mouth opening range greater than $35 \mathrm{~mm}$ was marked as M1 stage followed by M2 and M3 stages with mouth opening range between $25-35 \mathrm{~mm}$ and $15-25 \mathrm{~mm}$ respectively. However, the value less than $15 \mathrm{~mm}$ was graded as M4 stage.7 With the advancement of disease, there is high risk of malignant transformation leading to poor prognosis 


\section{Methodology}

This was a retrospective study conducted in Osmania General Hospital,Hyderabad,India. The cases were selected from E N T Outpatient department of Osmania General Hospital,Hyderabad, from January 2013 to December 2015 . An oral expressed consent was taken from the patients regarding participation and publication of data. The patients suffering from OSMF irrespective of age and gender were included in the study. The data including age, gender, and chief complaints were recorded in a specially designed proforma for the study. Clinically, the site and presentation of the lesion along with the associated features including mouth opening, burning sensation, dryness of mouth and speech difficulty were evaluated. The patient's record and the data was recorded in the proforma.

The collected data was entered and analyzed by SPSS statistic software version 20. Descriptive statistics was calculated for the variables.

\section{Results}

A total of 70 patients with OSMF reported to E N T Outpatient department of Osmania General Hospital, Hyderabad during the study period.

The patients were divided into 4 groups according to their age. The highest incidence was recorded in second decade of life which was about $73 \%(\mathrm{n}=51)$ followed by $21 \%(\mathrm{n}=15)$ of patients in 21-30 years of age group. However, only $3 \%(\mathrm{n}=2)$ of patients were recorded in extremes of age including both $0-10$ and 31-40 year age group. The average age of patients was 18 years.

Table 1.Distribution of patients according to age

\begin{tabular}{|c|c|c|}
\hline & Number of patients & Percentage \\
\hline $0-10$ & 2 & 3 \\
\hline $11-20$ & 5 & 7 \\
\hline $21-30$ & 1 & 2 \\
\hline $31-45$ & 2 & 3 \\
\hline & 70 & $100 \%$ \\
\hline
\end{tabular}

The data furthermore revealed that out of 70 patients in the study, $60 \%(n=42)$ were males and only $40 \%(\mathrm{n}=28)$ were females. This suggests higher incidence of disease in males. The male to female ratio was $1.5: 1$

Figure 1: distribution of patients according to gender.

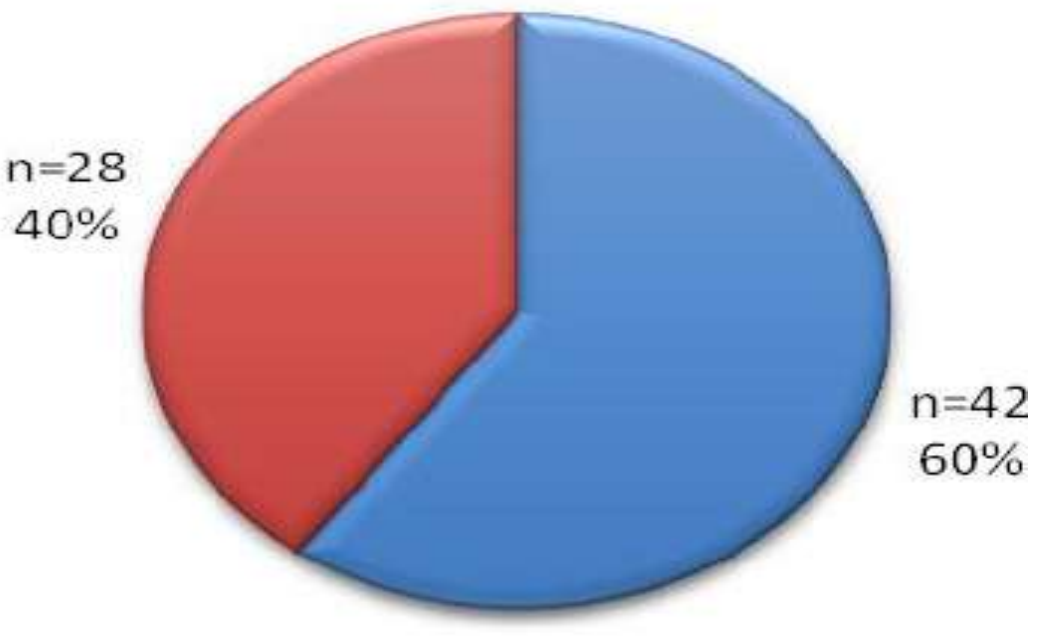

\section{Male Female}

Of all the patients reviewed, $97 \%(\mathrm{n}=68)$ of patients presented with burning sensation followed by $93 \%$ $(\mathrm{n}=65)$ of patients with complaint of limited mouth opening (Figure 2) and dryness of mouth. However, only $7 \%(\mathrm{n}=5)$ presented with vesicles and none of the patients complained of difficulty in speech. 
Figure 2. An OSMF patient with limited mouth opening

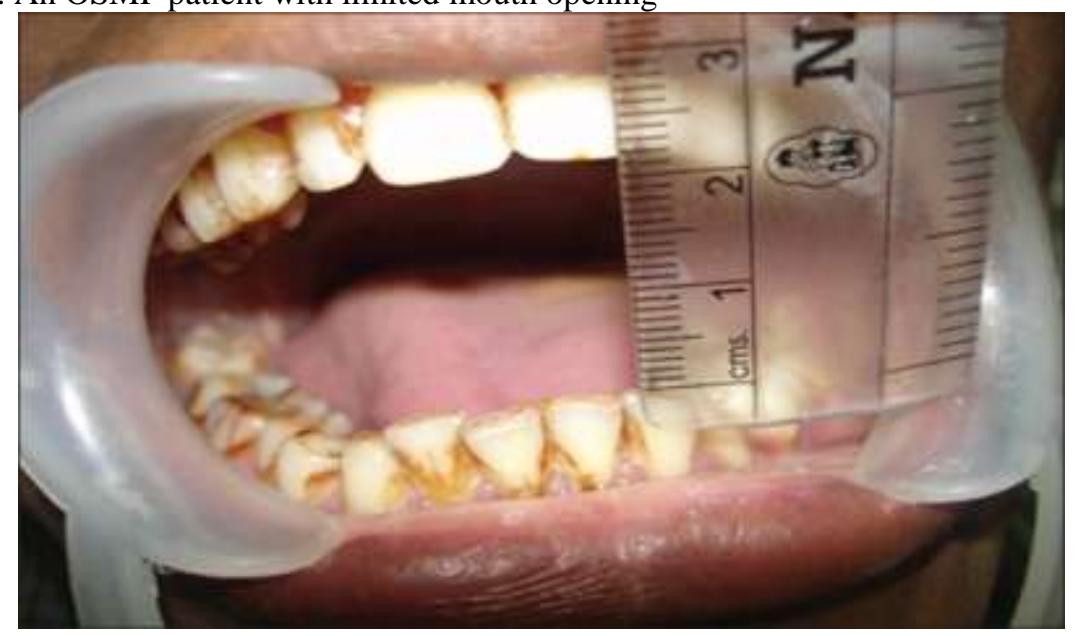

Figure 3.OSMF encountered on palate

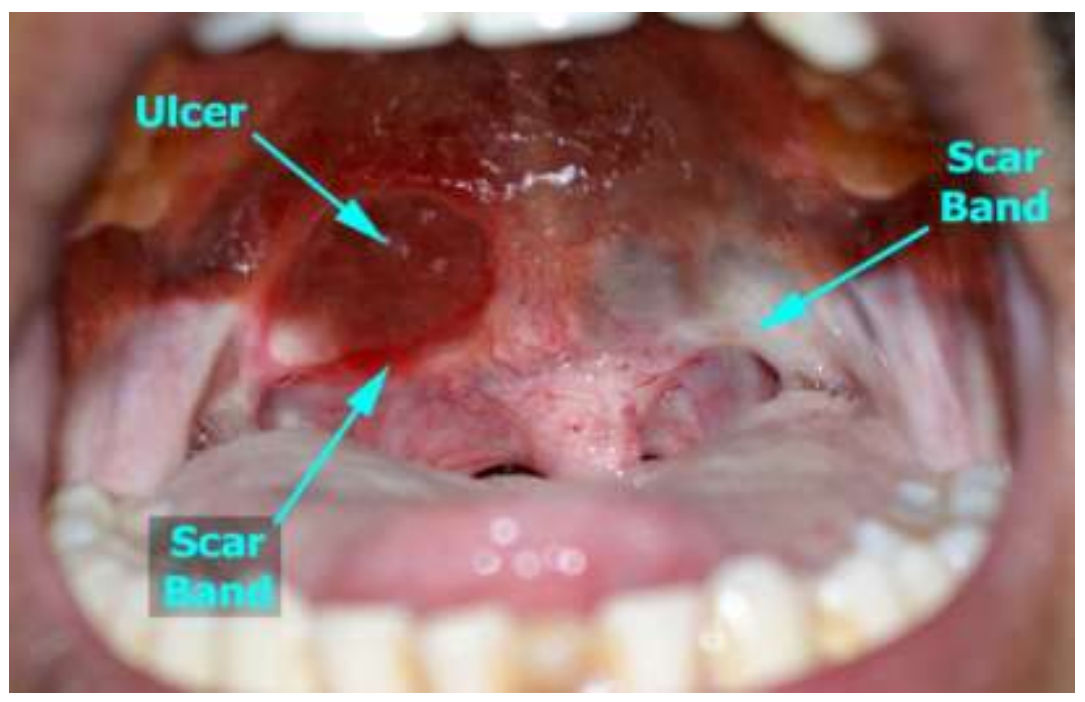

The lesion was predominantly encountered on palate with $73 \%(n=51)$ of patients evaluated for same (Figure 3). Moreover, 64\% $(n=45)$ and $57 \%(n=40)$ of patients were examined with lesion on buccal mucosa and lips respectively. Only 1 out of 70 patients presented with involvement of tongue as well.

Figure 4:distribution of patients according to site of OSMF 


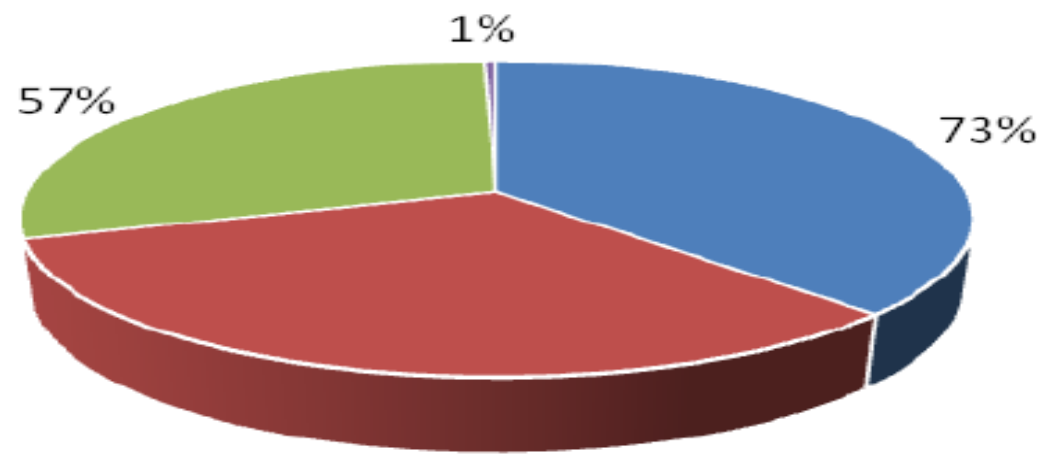

$64 \%$

- Palate - Buccalmucosa = Lips - Tongue

Figure 5. Frequency of limited mouth opening in OSMF patients

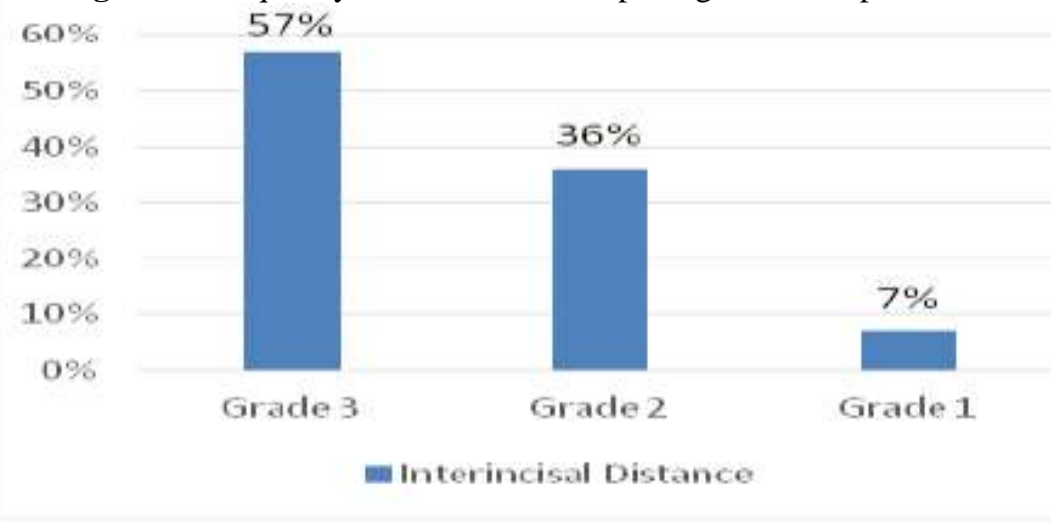

\section{Discussion}

Oral submucous fibrosis (OSMF) is a chronic precancerous condition of oral mucosa which is predominantly caused by excessive intake of areca nut. The data reveals that the disease is commonly found in younger age group i.e. from 11-20 years of age. In previous studies, OSMF was found to be more prevalent in a later age of life i.e. from 20-45 years of age.An eight fold increase has been observed since 1980s in the prevalence of OSMF in younger age group which is strongly related to increase popularity and availability of areca nut containing products. The advertisement and marketing strategies of these products forces the youth to accept it as sign of modernity and fashion. Gupta et al found in his studyrevealed that consumption of gutkha and tobacco to be popular among younger age group in Asian countries. He further supported his study by evaluating the Indian market of areca nut, tobacco, pan masala and gutkha which was rated for a worth of Rs. 25 billion.10 Furthermore, Oakley et al. in the region of Mariana Islands also found a higher rate of disease in teenagers which was correlated to increase consumption of areca nut preparation in their high school.11

A conclusive evidence for the pathogenesis of OSMF states that the disease is caused by different chemical constituents of areca nut and genetic susceptibility of the disease. These chemical constituents have been found to enhance the upregulation and/or degradation of collagen in the oral epithelium which leads to distortion of laminapropria and submucosa of oral cavity ${ }^{11}$. Different in vitro studies carried out on human 
fibroblasts using areca extract explains the role of copper in areca nut as a strong mediator of fibrosis. Recent advances also support the development and pathogenesis of OSMF with collagen related genes.

In this study, most of the OSMF cases were observed with a male preponderance. This is in accordance with previous reports from Hazarey et al., Yuh-Yuan et al., Merchant et al., Ali SM et al., Reddy V et al., and Ahmad MS et al. All these authors favorably support this study with an evidence that the consumption of areca nut is greater in men than women. According to a study conducted in Indian state of Wardah, the prevalence of gutka usage by men and women was documented to be $46.4 \%$ and $20 \%$ respectively. However, the studies reported by Joshi, Desa, and Sirsat and Khanolkar contradict these findings by documenting equal number of OSMF cases among both males and females. Moreover, Rao and Raju, Rao and Pindborg et al. reported a preponderance in females. These variations could be due to habit consumption of areca nut equally or predominantly by women in the tropical areas of study in that point of time.

The predominant complaint of OSMF patients in our study was burning sensation and limited mouth opening evaluated as $97 \%$ and $93 \%$ respectively. The results relates with previous studies ofShiau and Morawetz et al. who also concluded that majority of OSMF patients complaint of burning sensation followed by difficulty in mouth opening. The very first and significant feature of OSMF is stomatitis which results in epithelial atrophy of oral mucosa. The patient thus complains of burning sensation while eating hot and spicy food. Stomatitis can continue till all stages of OSMF. with the advancement of disease, fibrous bands appear in the mucosa which gradually become palpable. The severity of OSMF depends upon the number of oral sites involved and the amount of thickness and rigidity of mucosa.an important criteria in diagnosing the progression of the disease is by determining the inter-incisal mouth opening. Majority of the cases of OSMF reports with an increased severity.

\section{Conclusion}

Oral submucous fibrosis is a chronic debilitating disease that predominantly affects younger age group adults with male predilection. The condition is predominantly characterized by burning sensation and limited mouth opening. The majority of patients present with an increased severity of disease with grade 3 inter-incisal mouth opening which suggests lack of awareness among patients and delayed diagnosis. An advancement in diagnostic aids is required to arrest the disease in initial stages. Moreover, awareness campaigns must be carried out to educate the youth about the hazardous habits.

\section{References}

[1]. Schwartz J. AtrophiaIdiopathica (tropica) mucosaeoris. Demonstrated at the Eleventh International Dental Congress, London, 1952 (cited by Sirsat\&Khanolkar). Ind. j. med. Sci., 1962, 16: 189-197.

[2]. Joshi SG. Submucous fibrosis of the palate and pillars. Ind. j. otolaryn., 1953, 4: 1-4.

[3]. Pindborg JJ, Sirsat SM. Oral submucous fibrosis. Oral surg. oral med. oral pathol., 1966, 22: 764-779.

[4]. Lemmer J, Shear M. Oral submucous fibrosis: a possible case in a person of Caucasian descent. Br. dent. j., 1967, 122: 343-346.

[5]. Pindborg JJ, Singh B. Formation of vesicles in oral submucous fibrosis. Acta path. microbiol. scand., 1964, 62: 562-566.

[6]. Sirsat SM, Pindborg JJ. Subepithelial changes in oral submucous fibrosis. Acta path. microbiol. scand., 1967, 70: 161-173.

[7]. Wahi PN et al. Submucous fibrosis of the oral cavity: histomorphological studies. Br. j. cancer, 1966, 20: 676-687.

[8]. World Health Organization. Guide to epidemiology and diagnosis of oral mucosal diseases and conditions. Community dent. oralepidemiol., 1980, 8: 1-26.

[9]. Gupta PC et al. Incidence rates of oral cancer and natural history of oral pre-cancerous lesions in a

[10]. year follow-up study of Indian villagers. Community dent. oralepidemiol., 1980, 8: 287-333.

[11]. Rajendran R. Oral submucous fibrosis: etiology, pathogenesis and future research. Bull World Health Organ1964; 72(6):985-996.

[12]. Aziz SR. Oral submucous fibrosis: an unusual disease. J N J Dent Assoc. Spring 1997; 68(2):17-19.

[13]. Ranganathan K, Uma Devi M, Joshua E, Kirankumar K, Saraswathi TR. Oral submucous fibrosis: a case control study in Chennai South India. J Oral Pathol Med 2004;

[14]. Dyavanagoudar SN. Oral Submucous fibrosis: Review on Etiopathogenesis. J Cancer SciTher 2009; 1(2): 7277.

[15]. 5 Sultana N, Pallagatti S, Mohamed AI. P53 expressions in oral submucous fibrosis and oral squamous cell carcinoma. Int J Oral Maxillofacial Pathol 2011; 2(1):9- 14 .

[16]. Donoghue M, Smitha B. Clinical and histopathological evaluation of collagen fiber orientation in patients with oral submucous fibrosis. Journal Oral MaxillofacPathol 2011; 15(2):154-159.

[17]. More CB, Patel SH, Adalja C, Kamatchi V, Venkatesh R. Proposed clinical classification for oral submucous fibrosis. Oral Oncol 2012; 48: 200-202.

[18]. Angadi PV, Rap SS. Areca nut in pathogenesis of oral submucousfibrosis:revisited. Oral MaxillofacSurg 2011; 15:1-9.

[19]. Pindborg JJ, Metha FS, Daftary DK. Incidence of oral cancer among 30,000 villagers in India in a 7 year follow up study of oral precancerous lesions. Community Dent Oral Epidemiol. 1975; 86:88.

[20]. Gupta PC, Ray CS. Epidemiology of betel quid usage. Ann AcadMed Singapore 2004; 33:31-36. 